\title{
Novel Tandem Duplication in Exon 1 of the SNURF/SNRPN Gene in a Child with Transient Excessive Eating Behaviour and Weight Gain
}

\author{
S. Naik ${ }^{a, b}$ N.S. Thomas ${ }^{a, d} \quad$ J.H. Davies ${ }^{c} \quad$ M. Lever ${ }^{d} \quad$ M. Raponi ${ }^{a} \quad$ D. Baralle ${ }^{a, b}$ \\ I.K. Temple ${ }^{a, b} \quad$ A. Caliebe ${ }^{b}$ \\ ${ }^{a}$ Academic Unit of Genetic Medicine, Division of Human Genetics, University of Southampton, School of Medicine, \\ ${ }^{b}$ Wessex Clinical Genetics Service and 'Paediatric Endocrinology Department, Southampton University Hospitals \\ Trust, Southampton, dWessex Regional Genetics Laboratory, Salisbury Health Care Trust, Salisbury, UK
}

\section{Key Words}

Duplication in SNURF/SNRPN • Loss of SNURF expression •

Prader-Willi syndrome

\begin{abstract}
A deletion in $15 q 11.2$ involving the SNURF/SNRPN gene is the typical finding in patients with Prader-Willi syndrome. Apart from translocations disrupting this gene, no other mutation types have been described so far. We report a patient in whom a small duplication in exon 1 of the SNURF/SNRPN gene was diagnosed which is predicted to interrupt only SNURF expression. The patient was investigated due to overgrowth, increased appetite and developmental delay in childhood. This duplication was inherited from her father who carries the duplication on his paternal chromosome 15 and also had transient excessive eating behaviour as an adolescent. RNA studies showed that the duplication introduces a premature stop codon in SNURF.
\end{abstract}

Copyright $\odot 2012$ S. Karger AG, Basel

Prader-Willi syndrome (PWS) is a neurodevelopmental syndrome characterised by hypotonia, developmental delay and excessive appetite. The aetiology of PWS is het- erogeneous. In about $75 \%$ of patients a paternal deletion of the region 15q11.2 can be detected. Maternal uniparental disomy of chromosome 15 is seen in $20 \%$ of patients. Three percent of patients have an imprinting defect, which in some cases results from a mutation of the imprinting centre. No alterations are detected by current methods in about $2 \%$ of patients with a clinical diagnosis of PWS [Gardner and Sutherland, 2004].

A number of patients have been reported in whom either classical or atypical PWS was due to a translocation disrupting the SNURF/SNRPN region [Schulze et al., 1996; Sun et al., 1996; Kuslich et al., 1999; Wirth et al., 2001] or to an atypical deletion causing partial loss of the PWS critical region in 15q11.2 [Sahoo et al., 2008; Kanber et al., 2009].

Recently, Duker et al. [2010] reported a child with infantile hypotonia, early-onset morbid obesity, and hypogonadism with a paternally inherited microdeletion at 15q11.2 involving just the SNORD116 C/D box snoRNA cluster. This case provides evidence for the importance of this region in PWS.

To the best of our knowledge, no other mutations in SNURF/SNRPN have been described, except for disruption by translocation or deletions. Here we report a child with speech delay and excessive appetite resulting in

\section{KARGER}

Fax +4161306 1234

E-Mail karger@karger.ch

www.karger.com
(C) 2012 S. Karger AG, Basel

$1661-8769 / 11 / 0022-0076 \$ 38.00 / 0$

Accessible online at:

www.karger.com/msy
Dr. Swati Naik

Wessex Clinical Genetics Service

Princess Anne Hospital, Coxford Road

Southampton SO16 5YA (UK)

Tel. +44 238079 6170, E-Mail drswatinaik@ doctors.org.uk 
Fig. 1. A Proband at the age of $24 / 12$ years. Note the full face, indicative of the overweight. B, C Proband at the age of 11.5 years. Note the average build and tapering fingers. A, B The face shows hypertelorism, flat nasal bridge, bulbous nasal tip and epicanthic folds.
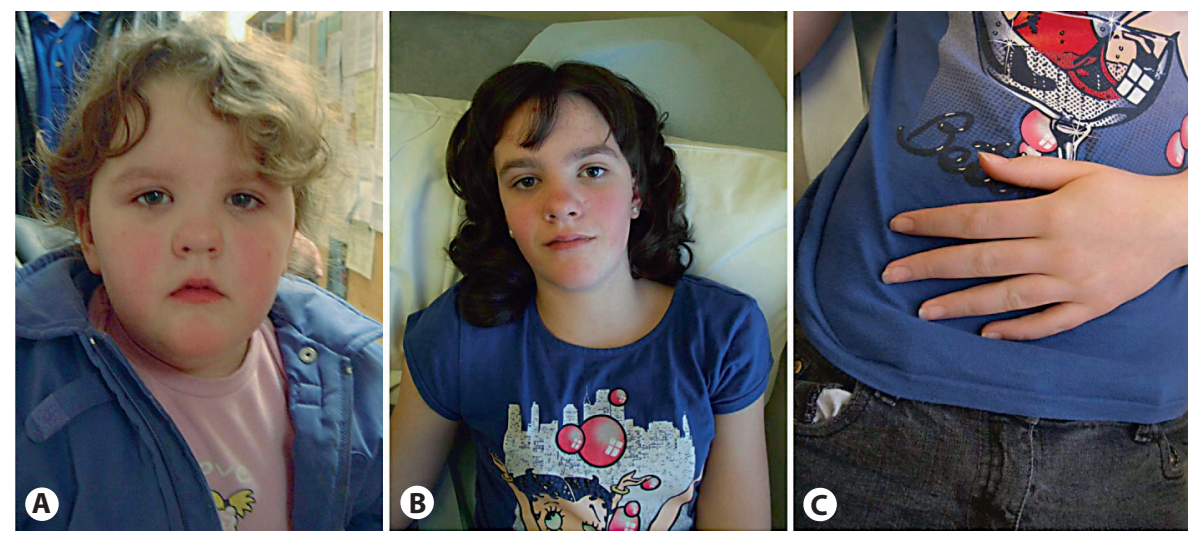

childhood obesity, who was found to have a paternally inherited 25-bp duplication in exon 1 of the SNURF/ SNRPN gene.

\section{Case Report}

The proband is the second child of a non-consanguineous Caucasian couple. Her mother was 30 years old and her father was 33 years old at the time of conception. The pregnancy was uneventful with normal antenatal scans. She was delivered by normal delivery at 38 weeks with a birth weight of $2.6 \mathrm{~kg}$ (25th centile). She was breastfed soon after birth, and there was no history of feeding difficulty. Her motor development was normal, and she started gaining weight in late infancy. Her parents raised their first concerns about her large size, speech delay and excessive weight gain at the age of 18 months. Endocrine investigations showed normal IGF1, IGFBP3, random GH and urinary GH and the cranial MRI was normal with no pituitary abnormalities. NSD1 sequencing was normal.

At the age of 2 years, the proband's height was $98.5 \mathrm{~cm}(+3.04$ $\mathrm{SD})$, the weight was $23.8 \mathrm{~kg}(+5.14 \mathrm{SD})$, and the head circumference was $51 \mathrm{~cm}(+2.13 \mathrm{SD})$. She had speech delay requiring speech therapy input. Her parents also reported behaviour abnormalities such as temper tantrums. On examination, her facial features revealed mild hypertelorism, flat nasal bridge, bulbous nasal tip and epicanthic folds (fig. 1A). The systemic examination did not reveal any abnormalities. At the age of 3 years her bone age was advanced by 1 year.

The growth parameters at the age of 11.5 years were: height 170 $\mathrm{cm}(+3.26 \mathrm{SD})$, weight $63.5 \mathrm{~kg}(+2.25 \mathrm{SD})$ and head circumference $57 \mathrm{~cm}(+2.69$ SD). She had mild learning difficulties and attended a special school. Presently, she could read and write and her behaviour had improved. Her eating was controlled by her parents, and the proband was successful in controlling overeating. On examination at this follow-up review, a tall build, a long tongue, tapering fingers and mild cubita valga were noted (fig. 1B, C).

The proband's father reported that at the age of 18 years he had weighed $108 \mathrm{~kg}$ and had significant problems resisting food. $\mathrm{He}$ described himself as having mild learning difficulties at school and dyslexia. However, as an adult he learnt how to control food intake and had a normal weight and height by his 30s.

\section{Laboratory Methods}

\section{Cytogenetic Studies}

Chromosome analysis was carried out on peripheral blood lymphocytes using standard techniques. For array CGH a $44 \mathrm{~K}$ oligonucleotide array (Agilent) was used.

\section{Molecular Studies}

Methylation-specific PCR (MS-PCR) for SNRPN exon 1 was performed according to Sun et al. [1996]. Genomic DNA was bisulphited using the EZ DNA Methylation Kit (Zymo Research), followed by a single tube MS-PCR assay employing a FAMlabelled common primer (5'-CTCCAAAACAAAAAACTTTAAAACCCAAATTCC-3') and 2 methylation-specific primers, one specific for the methylated allele (5'-TATTGCGGTAAATAAGTACGTTTGCGCGGTC- ${ }^{\prime}$ ) and the other specific for the unmethylated allele (5'-GTGAGTTTGGTGTAGAGTGGAGTGGTTGTTG-3'). Direct sequence analysis of SNRPN exon 1 was performed using the forward primer $5^{\prime}$-ACTGCGGCAAACAAGCAC- $3^{\prime}$. The reverse strand was sequenced using primer 5'-GGCCCAAATTCCGTTTATTC-3'. MS-PCR products and sequencing reactions were analysed on an ABI 3130. MS-PCR was analysed using Genemarker software and sequencing using $\mathrm{Mu}$ tation Surveyor software.

\section{Transcript Analysis}

Total RNA from the patient and 3 voluntary controls was isolated from whole blood drawn into PAX tubes (Qiagen). The tubes were stored at room temperature for $18 \mathrm{~h}$ after collection to maximise the RNA yield. Total RNA extraction was performed with the related PAX Blood RNA kit (Qiagen) according to the manufacturer's instructions. cDNA was synthesised from $1 \mu \mathrm{g}$ of RNA using the ImProm-II ${ }^{\mathrm{TM}}$ Reverse Transcription System (Promega) and PCR-amplified using the following primers: forward primer $5^{\prime}$-TGACGCATCTGTCTGAGGAG-3' in exon 1 and reverse 
primer 5'-ACTCCAATATGGCTTTAACCA-3' spanning exons 3 and 4 . The products were separated on a $2.5 \%$ agarose gel and visualised with ethidium bromide on a UV imaging system. Bands were excised from the gels, and the purified DNA fragments were sequenced.

\section{Results}

\section{Cytogenetic Studies}

Chromosome analysis revealed an apparently normal female karyotype, 46,XX. Array CGH did not show any imbalances.

\section{Molecular Studies}

Molecular testing for PWS showed that both maternal and paternal alleles were present at a normal dosage; however, the length of the paternal product was larger than expected. Sequencing of genomic DNA demonstrated that this was due to a tandem duplication of $25 \mathrm{bp}$ within exon 1 of the SNURF/SNRPN gene (fig. 2A). The duplication includes both the ATG transcription start site utilised by the SNURF gene and the splice donor site at the end of exon 1.

Follow-up analysis of the parents showed that the father carried the same abnormality and that this was also present on his paternally inherited chromosome 15 . The inheritance was deduced because the larger allele was the unmethylated allele (paternal). Subsequently, the duplication was shown to be absent from the proband's phenotypically normal sister, mother and paternal aunt. Grandparental samples were not available.

\section{Transcript Analysis}

The duplication results in 2 potential splice donor sites at the end of SNURF/SNRPN exon 1. Bioinformatic analysis using the BDGP splice site prediction program predicts that the duplication inactivates the original donor site and that instead the second donor site introduced by the duplication will be utilised. Analysis of the cDNA products revealed the presence of a larger, aberrant transcript of 289 bp in the patient compared with the 264-bp product from control cDNA. The results of the corresponding mRNA analyses are given in figure $2 \mathrm{~B}$. The $\mathrm{ab}-$ normal splice product was characterised by DNA sequencing, confirming the insertion of $25 \mathrm{bp}$ in the mature mRNA. This insertion causes a frame shift mutation with the introduction of a premature stop codon in SNURF (fig. 2C).

\section{Discussion}

We report a family with 2 members, father and daughter, both found to carry a small tandem duplication within exon 1 of the SNURF/SNRPN gene on the paternal chromosome 15. They both had a history of over-eating in childhood causing significant weight gain, but managed to control their eating behaviour later in life. Both had some learning difficulties, which appeared to be more severe in the daughter.

We are not aware of any other reports which describe sequence variation of just exon 1 of $S N U R F / S N R P N$. The duplication seen in this family was visible on MS-PCR, which is a widely used method for the detection of PWS. Therefore, it seems unlikely that it is a common variant, as it would have been described previously. The duplication alters the reading frame of the SNURF transcript, which utilises exons $1-3$, as the duplication introduces a premature stop codon. Since SNURF is normally expressed from only the paternal allele, no functional SNURF product will be present in carriers of the duplication. Both SNRPN and SNURF are transcribed from the same polycistronic mRNA, but transcription of SNRPN begins further downstream and is predicted to be unaffected by the duplication.

As the duplication is paternal in both carriers and segregates with the phenotype of over-eating, it seems possible it has a role in satiety. We acknowledge, however, that the finding in only 2 family members cannot be conclusive. Previous reports have addressed the clinical consequences of loss of SNURF/SNRPN expression by using phenotype-genotype correlations in patients with deletions and translocations of this gene.

Patients who had translocations disrupting only $S N U R F$ are very rare. We are only aware of 1 such patient, described by Sun et al. [1996], in whom a translocation disrupted SNURF. The patient had hypotonia, poor suck and developmental delay. He was also obese at the age of 6 months, and had an increased appetite from the age of 2 years. Length was normal for age. The translocation was of paternal origin and he was reported to have SNRPN expression. The patient had a different neonatal course with hypotonia and poor suck; however, the growth measurements were similar to those obtained for the patient presented in this report.

The patient described by Sahoo et al. [2008], who had a microdeletion sparing SNURF/SNRPN, had an almost classical PWS with over-eating, although normal height and head circumference. This is in line with the observation of de Smith et al. [2009], who reported a 19-year-old 
Fig. 2. A Sequencing of genomic DNA shows tandem duplication of 25 bp within exon 1 of the SNURF/SNRPN gene. B mRNA analysis. The abnormal splice product was characterised by DNA sequencing, confirming the insertion of 25 bp in the mature mRNA. C This insertion causes a frameshift mutation with the introduction of a premature stop codon in SNURF.

A Case Report of Duplication in Exon 1 of the SNRPN Gene
Exon 1...........AGGAGCGGTCAGTGACGCGATG GAGCGGGCAAGgtcagctgtgccggtggettctctc.........Intron 1

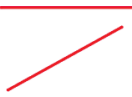

25 base pair duplication -

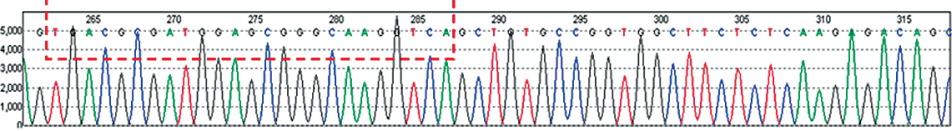
O.

A
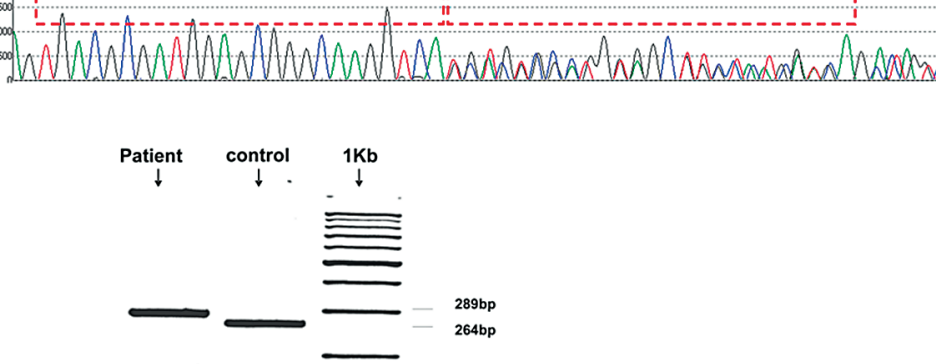

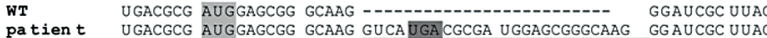

WILD TYPE SEQUENCE

GTCAGTGACGCGATGGAGC'AGGCAAGgtcagetgitgeeggtggettcteteaaga

]

DONOR SITE

SCORE 0.63

DUPUCATED SEQUENCE

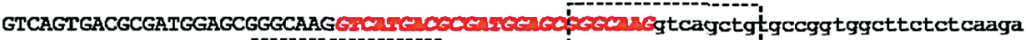

Original site score

now $<0.10$

\section{DONOR STE}

SCORE 0.63

\section{WILD TYPE SEQUENCE}

ucaggcggggaugugugcgaagccugccgcugcugcagcgagucuggegcAGAGUGGAGCGGCCGCCGG AGAUGCCUGACGCAUCUGUCUGAGGAGCGGUCAGUGACGCGAUGGAGCGGGCA AGgucagcugugecgguggcuucucuc

Donor site predictions

$$
\begin{array}{lll}
\text { Start } & \text { End } & \text { Score } \\
118 & 132 & 0.63
\end{array}
$$

Exon Intron

gggcaaggucagcug

\section{DUPLICATED SEQUENCE}

caggcggggaugugugcgaagccugccgcugcugcagcgagucuggegcAGAGUGGAGCGGCCGCCGGA GAUGCCUGACGCAUCUGUCUGAGGAGCGGUCAGUGACGCGAUGGAGCGGGCAA GGUCAUGACGCGAUGGAGCGGGCAAGgucagcugugecgguggcuucucuc
Donor site predictions

C

$$
\begin{array}{llll}
\text { Start End } & \text { Score } & \text { Exon Intron } \\
142156 & 0.63 & \text { gggcaaggucagcug }
\end{array}
$$


patient with severe hyperphagia and obesity and a microdeletion of only the HBII-85 snoRNA cluster. This makes it less likely that loss of SNURF/SNRPN is the only cause for over-eating in PWS, although it cannot be ruled out that alterations in this region contribute to a phenotype of over-eating. Furthermore loss of SNURF expression in the mouse is not associated with a definite phenotype [Tsai et al., 1999].

In summary therefore, there remains the possibility that a paternally inherited duplication in exon 1 of SNURF/SNRPN, which disrupts expression of SNURF, is responsible for the excessive appetite and obesity in the patient and her father. The phenotype of over-eating and learning problems segregates with the duplication. It will take more case reports of further patients with a comparable molecular phenotype to elucidate the role SNURF/ $S N R P N$ plays in PWS.

\section{Acknowledgement}

We thank Karin Buiting for expert discussion of the possible implications of the findings and encouragement to perform RNA studies on the patient.

\section{References}

de Smith AJ, Purmann C, Walters RG, Ellis RJ, Holder SE, et al: A deletion of the HBII-85 class of small nucleolar RNAs (snoRNAs) is associated with hyperphagia, obesity and hypogonadism. Hum Mol Genet 18:32573265 (2009).

-Duker A, Ballif B, Bawle E, Person R, Mahadevan $S$, et al: Paternally inherited microdeletion at $15 q 11.2$ confirms a significant role for the SNORD116 C/D box snoRNA cluster in Prader-Willi syndrome. Eur J Hum Genet 18: 1196-1201 (2010).

Gardner RJM, Sutherland GR: Chromosome Abnormalities and Genetic Counselling, 3rd ed. (Oxford University Press, New York 2004).

Kanber D, Giltay J, Wieczorek D, Zogel C, Hochstenbach R, et al: A paternal deletion of $M K R N 3, M A G E L 2$ and NDN does not result in Prader-Willi syndrome. Eur J Hum Genet 17:582-590 (2009)
Kuslich CD, Kobori JA, Mohapatra G, GregorioKing C, Donlon TA: Prader-Willi syndrome is caused by disruption of the SNRPN gene. Am J Hum Genet 64:70-76 (1999).

Sahoo T, del Gaudio D, German JR, Shinawi M, Peters SU, et al: Prader-Willi phenotype caused by paternal deficiency for the HBII$85 \mathrm{C} / \mathrm{D}$ box small nucleolar RNA cluster. Nat Genet 40:719-721 (2008).

Schulze A, Hansen C, Skakkebaek NE, Brøndum-Nielsen K, Ledbetter DH, Tommerup $\mathrm{N}$ : Exclusion of SNRPN as a major determinant of Prader-Willi syndrome by a translocation breakpoint. Nat Genet 12:452-454 (1996).
Sun Y, Nicholls RD, Butler MG, Saitoh S, Hainline BE, Palmer CG: Breakage in the SNRPN locus in a balanced $46, \mathrm{XY}, \mathrm{t}(15 ; 19)$ PraderWilli syndrome patient. Hum Mol Genet 5: 517-524 (1996).

Tsai TF, Jiang YH, Bressler J, Armstrong D, Beaudet AL: Paternal deletion from Snrpn to Ube3a in the mouse causes hypotonia, growth retardation and partial lethality and provides evidence for a gene contributing to Prader-Willi syndrome. Hum Mol Genet 8: 1357-1364 (1999).

Wirth J, Back E, Hüttenhofer A, Nothwang HG, Lich C, et al: A translocation breakpoint cluster disrupts the newly defined $3^{\prime}$ end of the SNURF-SNRPN transcription unit on chromosome 15. Hum Mol Genet 10:201-210 (2001).

ZZeschnigk M, Lich C, Buiting K, Doerfler W, Horsthemke B: A single-tube PCR test for the diagnosis of Angelman and Prader-Willi syndrome based on allelic methylation differences at the SNRPN locus. Eur J Hum Genet 5:94-98 (1997). 